\title{
Aspects of modelling classical or synchronous modelling with Solid Edge ST 9
}

\author{
Adrian Mihai Goanta ${ }^{1,}{ }^{,}$, and Georgeta Haraga $^{2}$ \\ 1"'Dunarea de Jos" University of Galati, Engineering and Agronomy Faculty of Braila, Research \\ Centre for Mechanics of the Machines and Technological Equipments, Calea Calarasilor Street, \\ 810017, Braila, Romania \\ 2"Politehnica" University of Bucharest, Department of Engineering Graphics and Industrial Design, \\ Splaiul Independentei No.313, Street, District 6, Postal Code 060042, Bucharest, Romania
}

\begin{abstract}
The current situation of the design activity is dependent on both the level of training of the human resources and the financial resources of companies required purchasing the design software packages and complex calculation equipment. Consequently, the situation is very diverse in the sense that there are design cases using only drawing software but also classical 3D or synchronous modelling situations, simple or integrated into software packages that meet the Product Lifecycle Management (PLM) principles. The natural tendency in modelling and design is primarily to the high computing power integrated software or somewhat simplified versions that, however, allow at least FEA modelling, simulation and the related $2 \mathrm{D}$ documentation. The paper presents some aspects of modernity in synchronous modelling as compared to the classic one, made with 2016 version of Solid Edge software from SIEMENS. Basically there were studied and analysed aspects of modelling ease, speed of changes and also optimization of commands in the modelling process of the same piece in the two versions mentioned: classic and synchronous. It is also presented the alternative path from one method to another within the same process of piece modelling, depending on the advantages provided by each method. In other words, the work is based on a case study of modelling a piece under the two modelling versions of which some aspects were highlighted and conclusions were drawn.
\end{abstract}

\section{Evolution of Traditional Design Techniques and State of the Art}

In small and medium business, traditional design before 1980 was done on board and paper followed by translation of both assembly and execution drawings on vellum transparent vellum/tracing paper which could be further multiplied in many copies and distributed in workshops for subsequent making and processing of the product. Original drawings on transparent tracing paper were archived in special libraries and archiving was based on the name and number assigned to each project. Any changes applied to older projects were

\footnotetext{
* Corresponding author: goanta_a_m@yahoo.com
} 
made by adding new drawing boards, either as assembly drawing or execution drawing and, when too many changes were necessary, the entire documentation of the initial project was made again and eventually another identification number was assigned. With the development and expansion of computer technology, meaning the emergence of PC computer systems and their more powerful version, called graphics workstations, work could move on gradually from the design to the drawing board to computer aided design in the sense of computer - based drawing. In high technology sectors with adequate funding, graphic stations began to be used for three-dimensional modelling to achieve a photorealistic visualization close to the real designed products. Graphics station hardware resources of that time were well above personal computers, which used to determine very high costs of acquisition for most design companies. One should not forget that the acquisition costs of computers were added to the costs of commercial licenses of the software. If we compare the computing systems of today with performance of the graphics workstations of those times, it is hard to believe how was possible to work $3 \mathrm{D}$ at that historical times at the beginning of three-dimensional designing, considering the fact that the processors had maximum $250 \mathrm{MHz}$ frequency and graphics cards, particularly important for 3D visualizations, did not exceed 4-8 MB AGP memory. Compared with those graphics workstations, personal computers of the time managed with great difficulty to generate a Wireframe of 3D solid and when they did it, they did it gradually giving the impression of a lengthy animation. At this point, it must be said that the situation is extremely varied in terms of the design method applied. This is because companies have extremely varied sizes and financial capacities. Thus at the current moment, small firms still use the method of computer -aided design while large business with extended activity and equally significant financial resources have implemented 3D design which implicitly allows getting automatic orthogonal or axonometric projections, the designer having just to add the necessary sizes for manufacturing, shape and position deviations, technical notes and all the other documents required by the rules of the technical drawing. It should be noted that at this moment there are companies that have managed to overcome this level of design complexity, meaning that they use integrated PLM software's (Product Lifecycle Management) which in a single file introduce information about both the 3D model, the execution drawing of the model, and information about the physical properties of materials, finite element analysis results, information on manufacturing simulation, etc.

\section{The classic method with tree-history (ordered)}

The tree - history method is also called parametric modelling method, where the 3D model is based on two-dimensional parameters and two-dimensional constraints that help the part keep its form according to the related parts family. At this point it is also called the classical method [1] because it tends to be generalized for all design firms. The moment for launching this method was 1989 when the company T-FLEX and a later the company ProEngineer introduced, as a working method in the field of CAD (Computers Aided Design), those 3D parameterized models, which implicitly kept the history of all operations that have led to obtaining three-dimensional model. Synthetically, the parametric modelling assumes, in addition to 3D modelling, the existence of defined parameters within a certain timeline. These parameters can be changed both during the design / modelling and at the end, but cause chain propagation of all the other parameters that are in a determination relationship with the changed one. Stages to be completed when using this method are: defining the sketch plan, sketch achievement in the drawing plan chosen, making parameterized listing, imposing 2D restrictions, defining 3D characteristics and combining them. Regardless of the method chosen (classical or synchronous) the design continues with orthogonal and axonometric projections, projections sizing, adding shape and position 
deviations, and adding technical notes. For Solid Edge this tree- history method is called ordered. Basically, in the area of the modelling screen, superimposed on it there is an area where a number of information are written about the history of all operations performed, which is called PathFinder. For example Figure 1 illustrates the 3D models related to two pieces with different tree histories, but both contain at PathFinder level the same categories of information: file name, references of the base plans, the label "Ordered" and then a set of sketches followed by designation of a 3D feature. Mention must be made that in the files chosen it was considered that sketches should not be visible not to make difficult the understanding of the final geometry.

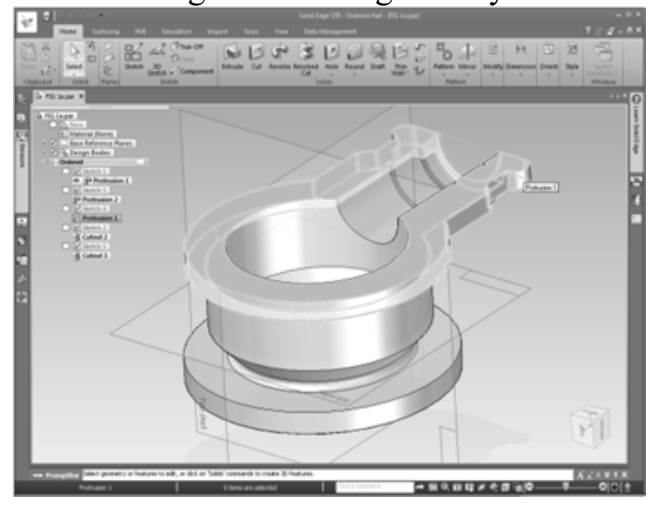

$\mathrm{a}$

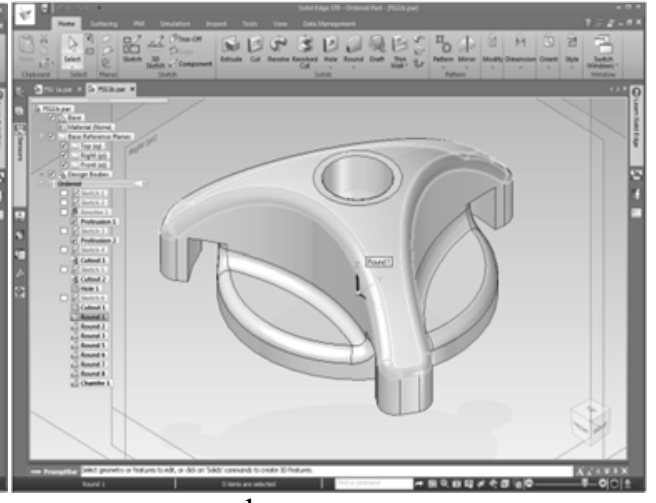

b

Fig. 1. Parts made with (ordered) tree history.

Also it can be seen in each of the examples in Figure 1 that selecting a 3D feature from the tree PathFinder involves the allocation of a certain colour (shade of gray) to those portions of the three-dimensional model which relates to the feature selected. The tree structure of the PathFinder also observes the chronology of generating sketches or local 3D feature. Changing any element of the PathFinder structure means to keep in the modelling space those items inside the selected item for editing and not showing temporarily the subsequent ones [2].

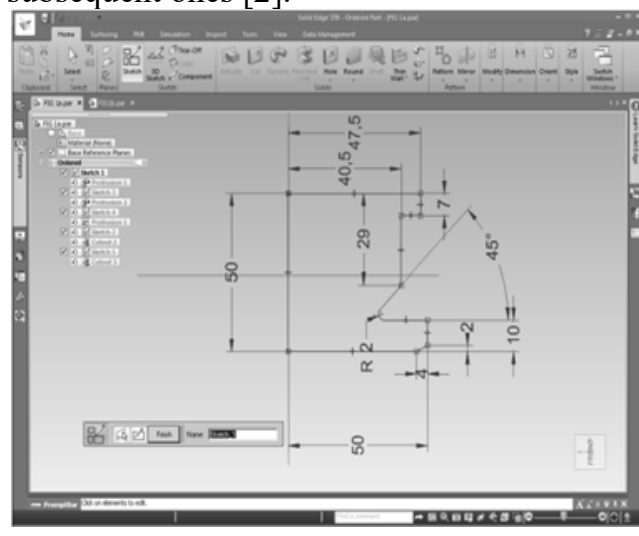

a

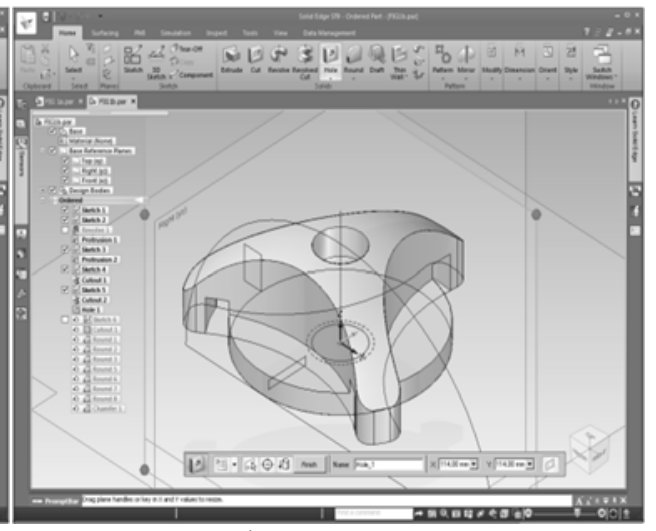

b

Fig. 2. Editing components from the tree structure.

As seen in Figure 2, the sketch editing involves its viewing in the modelling space and temporary not showing all the elements of the tree structure achieved subsequently. Upon completion of editing by clicking "Finish", the sketch will close and will involve changes related to the characteristic "Protrusion1" while updating all the characteristics not shown 
during editing. Figure $2 \mathrm{~b}$ shows that editing the feature „Hole1" involves viewing all previous features and temporary concealment of those subsequent to the edited one. In other words in the chronology of the completed operations there are elements of "parent" type and elements of "child" type. In some cases, an item "parent" may have subordinated several elements of "child" type. During editing a "parent" item, all the elements of "child" type will be temporarily hidden. For decades modelling based on history ruled the CAD for various reasons among which: the undeniable superiority compared to the previous stage of drawing assisted by computer, making quick changes by editing parameters, applying the concept "Rolling back", the special utility of the "parent-child" relationship, etc.

\section{The Synchronous method}

The emergence of the Synchronous technology is in year 2008, when Siemens launches the Solid Edge version called ST1 (Synchronous technology) designed to achieve a true revolution in the design field as, along with the method of history modelling, introduced a new method and modality of editing solids. This new method has proved from the very beginning extremely useful especially in case of importing [3] in Solid Edge native files of other competing software. Until now continuing the modelling on such files imported from other software was difficult and time consuming. Synchronous technology is the combination of the best features based on CAD history and best functions of Direct Modelling, along with some special features, with the ultimate goal to speed up the completion of the model and making changes. This idea is suggested in Figure 3.

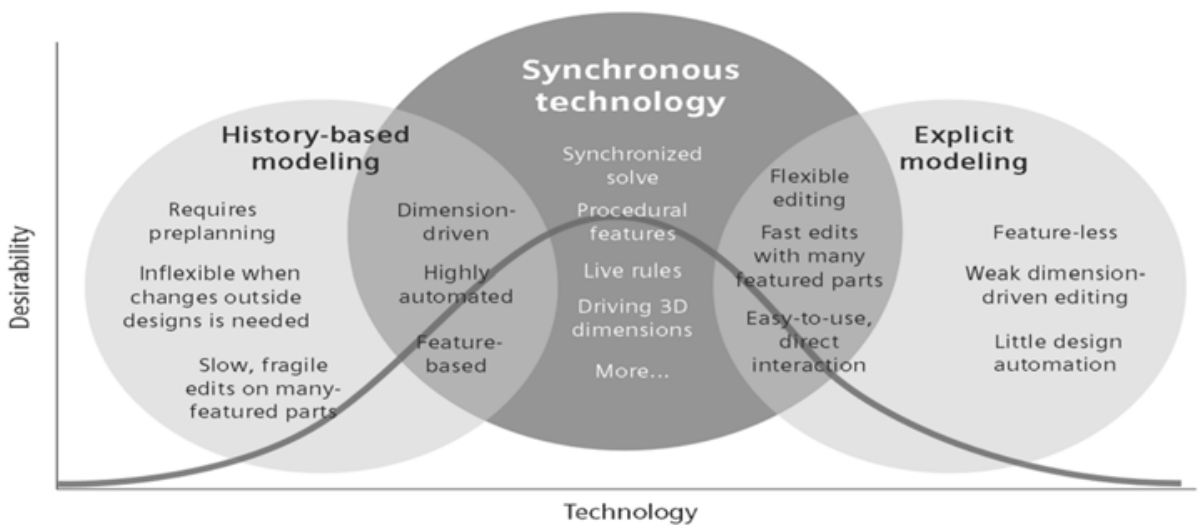

Fig. 3. How synchronous technology combines direct editing with parametric modelling [4].

Unlike the technology based on history, where things happen in turn, the synchronous version brings a total novelty according to which several processes occur simultaneously. The instruments that initialize these simultaneous processes are: PMI 3D Dimensions, Steering Wheel editing interface, Face Relations and Design Intent.

\subsection{PMI (Product Manufacturing Information's) 3D Dimensions}

In the classical variant with tree-history, changing the 3D feature is based on the link between "parent" and "child", meaning it acts on the parent who is actually a sketch and when the sketch is completed, the change is made on the "child" of the $3 \mathrm{D}$ feature type. This process is a sequential reconstruction. Unlike this case, the synchronous method offers the designer a new tool called PMI (Product Manufacturing Information) particularly strong 
and fast because it causes the change through direct action on the dimensions displayed on the screen, due to the fact that they no longer are to be found at the sketch, they are actually moved in the three-dimensional solid. Thus, the synchronous method causes the parentchild link to break but also add this new changing instrument called PMI that, when selecting a size of the solid, enables the designer to intervene directly on the solid by requiring application of the modification to the left, centre or right side, as shown in Figure 4.

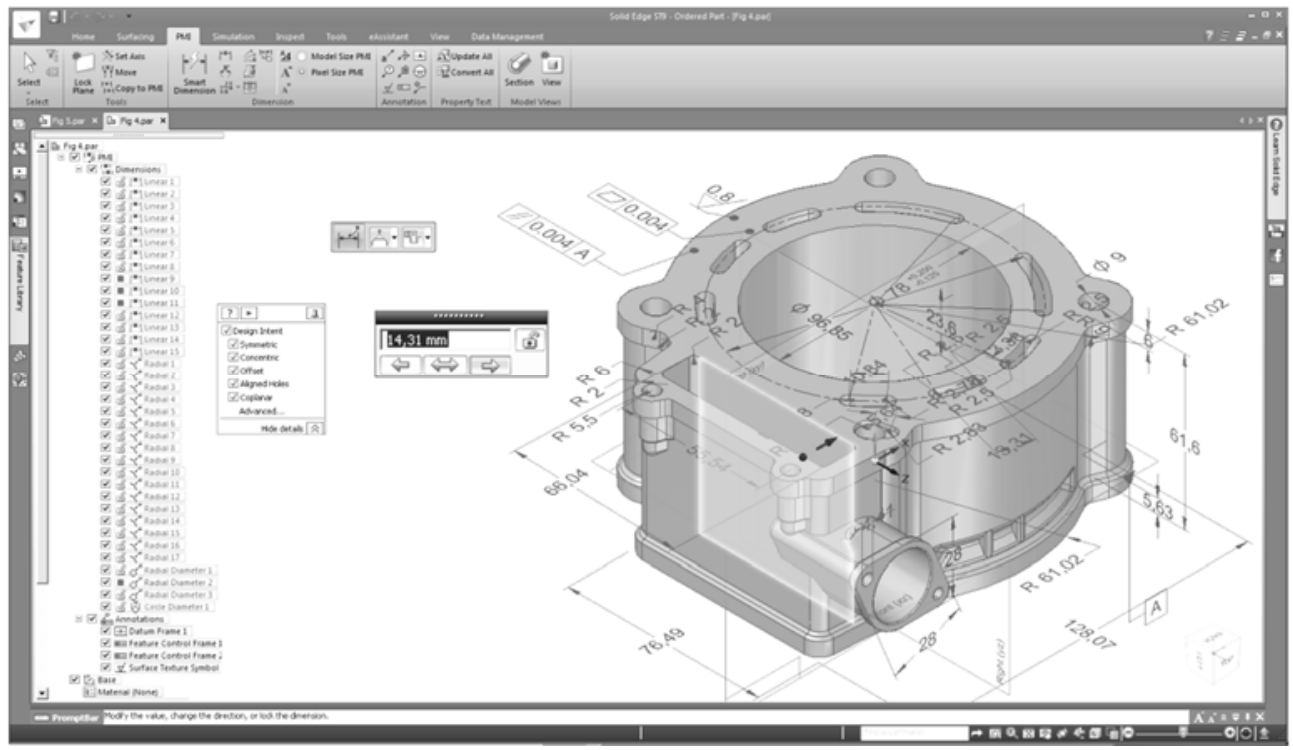

Fig. 4. Solid Edge PMI editor dialog.

At the same time it enables blocking the dimensional value by selecting the lock in the new working window.

\subsection{Steering Weel}

The steering wheel is an extremely powerful tool made available to the designer by the synchronous technology, by which one may select a particular surface, followed by its orientation in space and the choice of how to implement the change, meaning selecting a travel axis or the plan containing the tor axis of rotation. This tool determines the movement or rotation of the following elements: Reference planes (except the base reference planes) Coordinate systems (except the base coordinate system), Sketches, Sketch elements, Curves, Faces, Features and Design Bodies. The appearance of the steering wheel on the screen in the full version is a progressive one, meaning that from the first click on any of the model face it will be selected the surface and in that place only the origin and normal axis will appear, while the second click performed on origin will cause full appearance which will allow commencement of the orientation process in 3D space.

Progressive display of the wheel depends on the type of surface selected and the displayed elements within it are sufficient to achieve all the modifications possible for that surface.

\subsection{Face Relations and Design Intent}


In synchronous technology relationship between adjacent faces is no longer related to the parent-child relationship. This allows the system to simultaneously solve the model and eliminates the need for a sequential history reconstruction. If, for instance, a line in space is tangent to an arc, and the two graphic elements are coplanar, then the tangent relationship is inflicted on the two sides as well. In case of importing geometry from other 3D design software, the synchronous technology allows the user to add additional information to imported geometry. Design Intent and Advanced Design Intent panel are two tools that together reveal the true power of the synchronous technology. Design Intent dialog box displays the current rules which were associated with a selected geometry. The second level of help is Advanced Design panel. Here, users have full access to see what rules the system has applied for the selected geometry and all the rules on which can intervene to select them.

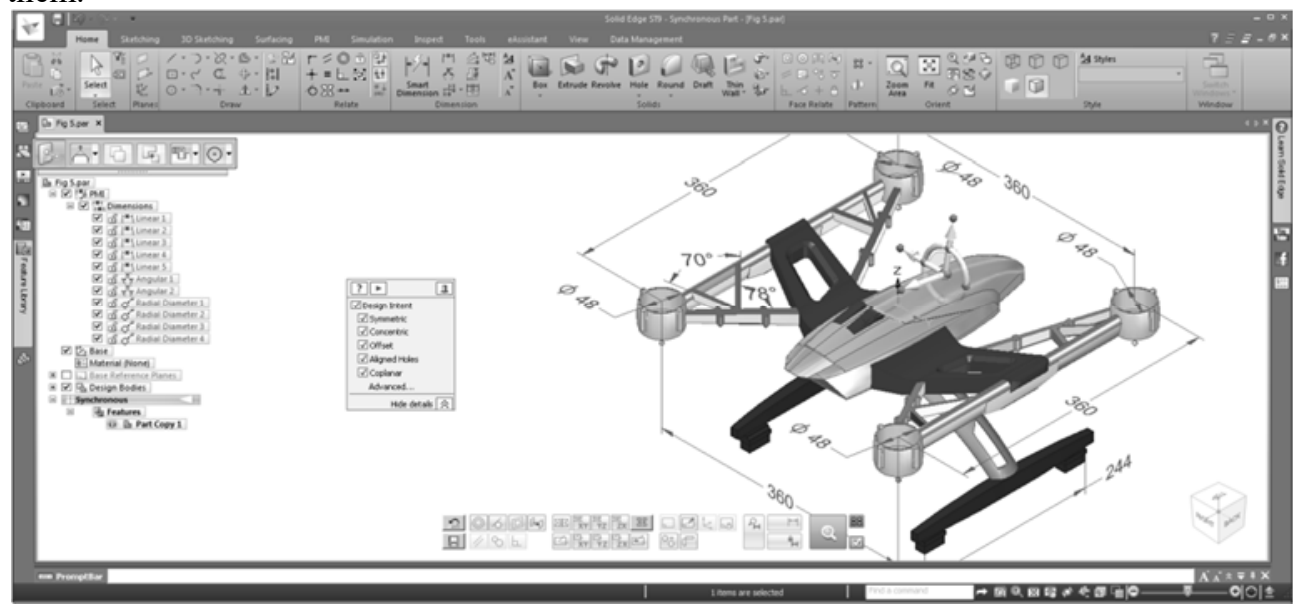

Fig. 5. Design Intent.

Rules in the lower region of Figure 5 may be enabled or disabled. There are several different options available to users, including control deactivation or stopping all controls.

\section{Conclusions}

Analysing the references mentioned in the end and those presented in this paper we can draw the following conclusions about the two methods studied, namely the ordered (with history) type and the synchronous type:

- The classic method (with history) has been applied for more than 25 years in the field of aided design and therefore has created stronger dependent relations among its users, who because of habit, prefer to apply a very well-known method rather than to test and then apply a superior method as the synchronous one.

- The classical method characterized by parent-child relationships that determine the sequential reconstruction of the model with every change of the parent is very much like the instruction lines chronologically ordered within any program.

- Both methods have strengths but also weaknesses, which is why it is recommended to be used with the aim of creating as robust models as possible. ST9 Solid Edge allows switching from one working mode to another whenever the user wants to. 
- The synchronous method has several extremely powerful tools such as: PMI 3D Dimensions, Steering Wheel editing interface, Face Relations and Design Intent, which have no correspondence with the history based method.

- The synchronous methods involve working with regions and require greater computing power from the hardware.

\section{References}

1. P. Dumitrache, JIDEG, 10/1, 5 (2015)

2. G. Musca, Solid Edge the complete solution for the projection mechanic, (Edp. PIM 2008)

3. A.M.Goanta, IOP Conf. Series: Mat. Science and Eng., Modern Technologies in Industrial Engineering, 145, 1 (2016)

4. https://www.plm.automation.siemens.com/en/products/solid-edge/st9/ , accessed by 10.01.2017 